\title{
Behavior of Wave Field on Graphite Surface Observed Using Reflection High-Energy Electron Diffraction Technique*
}

\author{
Yoshimi Horio ${ }^{\dagger}$ and Ryo Yamazaki \\ Department of Electrical and Electronic Engineering, Daido University, \\ Takiharu-cho 10-3, Minami-ku, Nagoya 457-8530, Japan \\ Junji Yuhara \\ Department of Energy Engineering, Nagoya University, Furo-cho, Chikusa-ku, Nagoya 464-8603, Japan
}

Yuji Takakuwa

Institute of Multidisciplinary Research for Advanced Materials, Tohoku University, Katahira 2-1-1, Aoba-ku, Sendai 980-8577, Japan

Masamichi Yoshimura

Surface Science Laboratory, Toyota Technological Institute, Hisakata 2-12-1, Tempaku-ku, Nagoya 468-8511, Japan

(Received 27 November 2017; Accepted 6 February 2018; Published 18 April 2018)

\begin{abstract}
Using reflection high-energy electron diffraction (RHEED), we demonstrate the behavior of a wave field formed on a graphite crystal surface. The sample used is highly oriented pyrolytic graphite, which is a polycrystalline (mosaic crystal) with a few micrometers-sized domain rotated on a plane. One-beam dynamical calculation is proved to be effective for analyzing the distance between the atomic planes. One-beam dynamical calculation is also used to obtain a wave field, which is incident electron density distribution, for surface normal direction. The characteristic behavior of the wave field at Bragg conditions is demonstrated by the Auger electron intensity of carbon excited by RHEED incident electron beam. This can be measured by changing the glancing angle using beam rocking Auger electron spectroscopy (BRAES). It has been found that the anomalous intensities in the measured BRAES profile almost correspond to the calculated wave field intensities on the atomic planes. [DOI: 10.1380/ejssnt.2018.88]
\end{abstract}

Keywords: Electron diffraction; RHEED; Auger ejection; Surface relaxation; Graphite

\section{INTRODUCTION}

The incident electron density distribution is referred to as the wave field behavior, which is investigated by measuring the Auger electron intensity while changing the incident glancing angle of reflection high-energy electron diffraction (RHEED). Here, this method is named as the beam rocking Auger electron spectroscopy (BRAES). It is considered that Auger intensity anomalies are caused due to the high-density wave field on the atoms. Previously, we investigated the wave field on a crystal surface, in particular under surface wave resonance (SWR) [1-6], when the wave field is not only concentrated on the surface region but also when it forms a standing wave parallel to both the surface and the incident azimuth. Secondary electron emission and Auger electron emission at resonance condition have been investigated based on the density distribution of the incident electron by Marten and Meyer-Ehmsen [7]. Surface normal wave-field distribution has also been studied by Kawamura and Maksym for explaining the effect of surface reconstruction on secondary electron emission distribution [8]. BRAES profile due to the wave-field distribution normal to a layered crystal surface such as graphite has not been studied yet on one-beam condition at which the standing wave parallel to the surface is averaged. It is interesting to know how the wave-field is distributed perpendicularly to the

\footnotetext{
* This paper was presented at the 8th International Symposium on Surface Science, Tsukuba International Congress Center, Tsukuba, Japan, October 22-26, 2017.

† Corresponding author: horio@daido-it.ac.jp
}

graphite surface on Bragg conditions. Research on twodimensional (2D) crystals [9-11] has intensified recently in both academia and in industries. In particular, graphite intercalations in $2 \mathrm{D}$ crystals are expected to add to their functionality. Basic research on the relation between the BRAES profile and the calculated wave-field distribution will contribute to the evaluation of the interlayer distance and the intercalated elements.

Anomalous X-ray intensities resulting from RHEED incident electron beam at Bragg reflection conditions were originally studied by Miyake et al. [12]. Yamanaka et al. demonstrated that anomalous X-ray emission could be explained by dynamical calculations under the one-beam condition [13]. In this study, we pay attention to the change of the Auger intensity excited by the wave field of RHEED while changing the incident glancing angle. Furthermore, since the wave field behavior constructed along the surface normal direction for a $2 \mathrm{D}$ crystal is unexplored, we investigate the relation between the BRAES profile and the diffraction condition.

There are two types of graphite crystals: natural graphite crystals, such as kish graphite or the natural graphite crystal of Sri Lanka; and artificial graphite crystals, such as highly oriented pyrolytic graphite (HOPG). Natural crystals are single crystals, however, they are inadequate for RHEED observation because their size and/or flatness may not be appropriate. On the contrary, artificial graphite crystals are adequate for RHEED observation; however, they are polycrystalline so that many small domains rotate in-plane in a disorderly fashion like a mosaic crystal.

In this study, we adapt the HOPG crystal as a sample. In this sample, the incident azimuth of RHEED is 


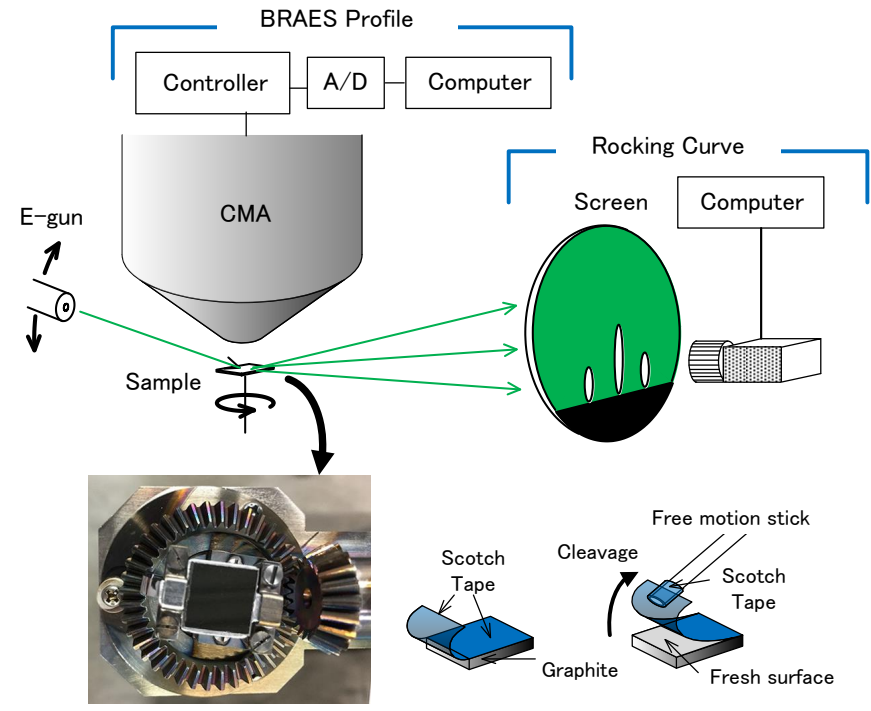

FIG. 1. Schematic diagram of the RHEED-AES apparatus and the top view of a graphite sample which is rotatable around the axis that is normal to the sample surface. Scotch tape and free motion stick were used for cleavage in vacuum.

assumed to be random because of the above-explained mosaic-like behavior of the crystal. Therefore, we use the average atomic potential of the plane, and the one-beam condition is assumed to have been satisfied. The rocking curves and the BRAES profiles of the graphite surface were measured and analyzed using one-beam dynamical calculations. Our results indicate that the Auger intensity anomalies are related to the wave field intensities that are located on the atomic planes of the graphite.

\section{EXPERIMENTAL}

The experiment was carried out using the RHEED apparatus combined with Auger electron spectroscopy (AES) under a base pressure of $1 \times 10^{-9}$ Torr; methodological details are described in Ref. [14]. A cylindrical mirror analyzer (CMA) was set above the sample surface to detect Auger electrons. In order to change the incident glancing angle of the electron beam, the electron gun was controlled mechanically with a $0.01^{\circ}$ resolution. The RHEED rocking curves were measured between $0^{\circ}-6^{\circ}$ at a $0.05^{\circ}$-interval using a charge-coupled device (CCD) camera and a computer system for data accumulation. At the same time, AES was also measured by the same incident electron beam of RHEED. In this manner, the Auger electron intensity in derivative mode could be obtained while changing the incident glancing angle, and thus the BRAES profile was obtained.

The used sample was a Panasonic Graphite Crystal (PGCX05) with a size of $12 \times 12 \times 1 \mathrm{~mm}^{3}$. The averaged domain size was a few micrometers and the mosaic spread was $0.51^{\circ}-0.60^{\circ}$ in specification.

Just prior to putting the sample in the RHEED-AES chamber, it was cleaved in atmosphere to expose the fresh surface. Even using quick cleavage treatment, the contaminant oxygen was slightly detected by AES and the background intensity of the RHEED pattern was rela-
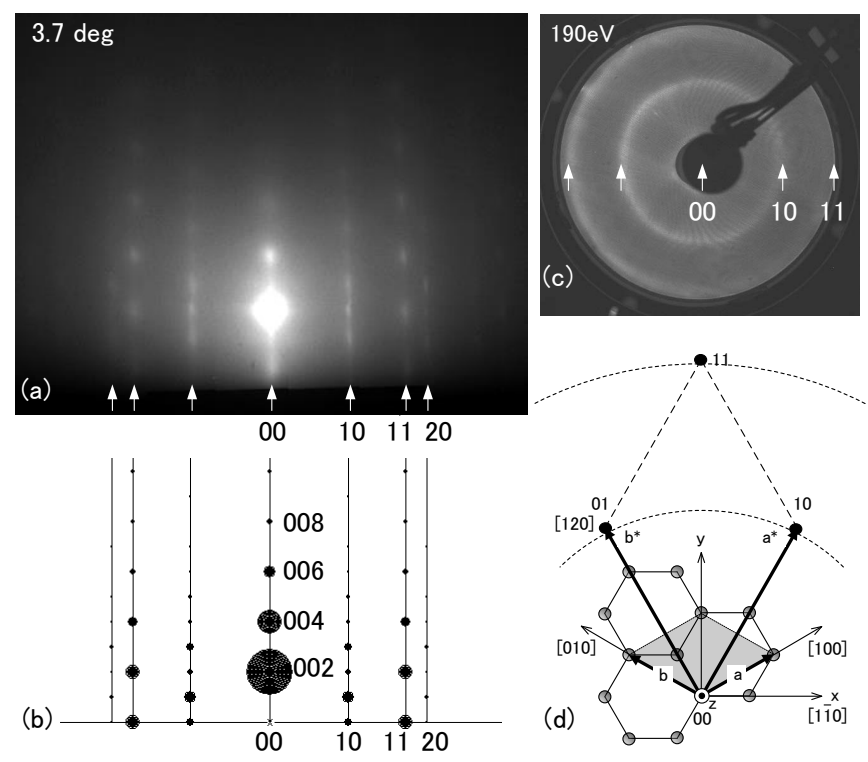

FIG. 2. (a) RHEED pattern taken from a graphite surface using a $10-\mathrm{keV}$ incident beam, (b) overlapped structure factors in $0^{\text {th }}$ Laue zone for $\langle 110\rangle$ and $\langle 100\rangle$ incident azimuths, which are indicated by the closed circles that are proportional to their intensities, (c) LEED pattern produced by a $190-\mathrm{eV}$ incident beam and (d) relation between the real and the reciprocal lattices with in-plane rotation.

tively high. In this study, therefore, cleavage was performed in a vacuum chamber using both of scotch tape and a free motion stick, as depicted in Fig. 1. In this case, the Auger peak of the contaminant oxygen was not detected at all. In comparison with air-cleaved surface, the RHEED pattern of vacuum-cleaved surface had a relatively high contrast.

\section{RESULTS AND DISCUSSION}

Figure 2 shows (a) a RHEED pattern of the vacuumcleaved graphite surface taken at an arbitrary azimuth, and (b) reciprocal points and rods in the $0^{\text {th }}$ Laue zone for $\langle 100\rangle$ and $\langle 110\rangle$ incident azimuths, where each spot area is proportional to the intensity of the crystal structure factor. The graphite extinction rule [Fig. 2(b)] agrees with the experimental RHEED pattern [Fig. 2(a)]. It is recognized that the experimental RHEED pattern consists with superposition of the reciprocal points and rods for these incident azimuths, which indicates that the surface domains rotate randomly in-plane. The low-energy electron diffraction (LEED) pattern in Fig. 2(c), which was taken from the same sample surface, shows concentric circles related to the inner 10 and outer $11 \mathrm{rod}$ reflections. The reflection indices are shown in Fig. 2(d) with the relation between the real and the reciprocal lattices. RHEED and LEED patterns show that the sample is a mosaic crystal, where reciprocal rods such as 10 or 11 are rotated around the center 00 rod and then the concentric cylinder is formed in the reciprocal space. RHEED and LEED patterns correspond to the intersection of the concentric cylinder and Ewald sphere.

For such a mosaic crystal, the one-beam dynamical 


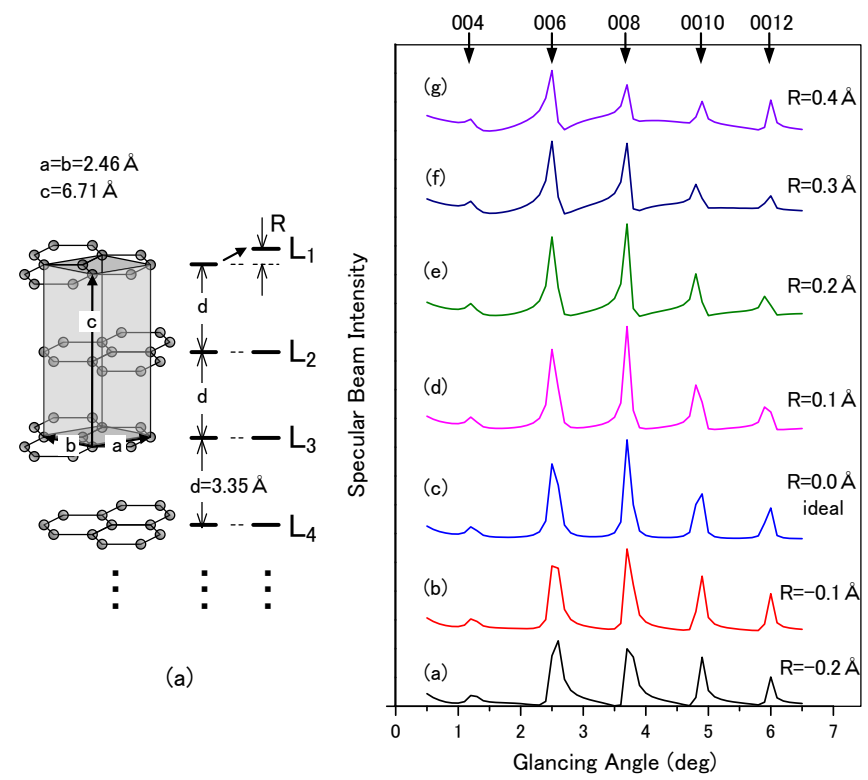

(b)

FIG. 3. (a) Relaxation parameter $R$ denotes the shift of the height of the first layer $\left(\mathrm{L}_{1}\right)$ from that of the ideal one. The positive and negative values indicate the expansion and the compression, respectively. (b) The calculated rocking curves changing the relaxation parameter $R$.

method [15] is considered effective for structural analysis because the crystal potential is averaged in plane. Then, only the surface normal periodicity can be focused on. The crystal potential deduced by Doyle and Tuner [16] is used. Here, the first layer $\left(\mathrm{L}_{1}\right)$ is assumed to be relaxed. The relaxation is expressed by a parameter $R$ which denotes the deviation of the inter layer distance between the first $\left(\mathrm{L}_{1}\right)$ and the second $\left(\mathrm{L}_{2}\right)$ layers from

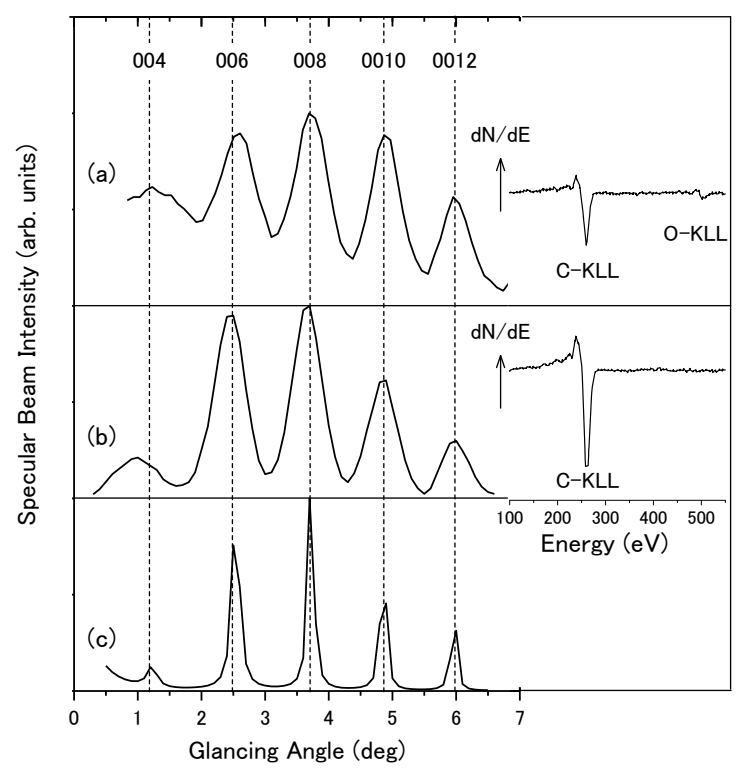

FIG. 4. (a) and (b) are the experimental rocking curves for the graphite surface cleaved in atmosphere and in vacuum, respectively. (c) is the calculated one for the ideal surface. Inset shows the Auger spectrum of each sample surface. the bulk one. Figure 3 shows the seven calculated rocking curves with $10-\mathrm{keV}$ incident beam for compressed relaxation $(R=-0.2 \AA$ and $-0.1 \AA)$, extended relaxation ( $R=0.1-0.4 \AA)$, and ideal $(R=0.0 \AA)$ structure models. It is observed that the shoulder appears in the high-angle sides of the Bragg peaks as the compression advances, and it appears in the low-angle sides for the expansion. The relative intensities of the Bragg peaks also change depending on the surface relaxations.

Figure 4 shows rocking curves of a specularly reflected electron beam using a 10-keV incident beam; Figs. 4(a) and $4(\mathrm{~b})$ are the experimental curves taken from a cleaved surface in atmosphere and in vacuum, respectively, and Fig. 4(c) is the calculated curve by the one-beam dynamical method based on the ideal structure, which is the same as in Fig. 3(c). It is observed that the experimental rocking curves in Fig. 4(a, b) reveal simple Bragg peaks similar to the calculated rocking curve in Fig. 4(c). The experimental rocking curve of Fig. 4(a), however, has relatively high background intensity, which is attributed to the presence of randomly adsorbed contaminant oxygen or carbon as shown in the inset of Auger spectra. The Bragg peaks of the calculated rocking curve are too sharp compared with that of the experimental ones because divergence effects, such as the incident beam spread and the mosaic spread, have not been considered. In the final analysis, $0.3^{\circ}$-divergence is taken into account in order to represent Bragg peak broadening and BRAES profile broadening. It is proved that the calculated rocking curve based on the ideal surface structure $(R=0.0 \AA)$ with no relaxation correlates well with the experimental one. These results indicate that the rocking curve analysis is a promising technique for evaluating inter-layer distances, especially for 2D layered material with van der Waals interaction that are difficult to estimate using density functional theory (DFT) calculations.

Figure 5 shows the calculated wave fields in the region from vacuum to the fourth layer $\left(\mathrm{L}_{4}\right)$ depending on the incident glancing angle at that moment while changing from $0.5^{\circ}$ to $6.5^{\circ}$. The inter layer distance between $\mathrm{L}_{n}$ and $\mathrm{L}_{n+1}$ is $c / 2$, where $c$ is the lattice constant $(6.71 \AA)$. It is observed that the amplitudes of the standing waves increase at the Bragg conditions $004\left(\theta=1.2^{\circ}\right), 006$ $\left(\theta=2.5^{\circ}\right), 008\left(\theta=3.7^{\circ}\right), 0010\left(\theta=4.9^{\circ}\right)$, and 0012 $\left(\theta=6.0^{\circ}\right)$. The number of standing-wave nodes between the nearest neighbor layers is $m$, where $2 m$ is a value of the third index of Bragg reflection $002 \mathrm{~m}$. The wave field is periodic in all the inter layers. It should be noted that the number of node looks like one for the low index 004 Bragg reflection. This exception is caused due to the superposition of two wide waves in an interlayer.

Attention should be devoted to the behavior of the wave field on each atomic layer and also to the Auger intensity that is excited by the wave field. Figure 6 shows the behavior of the calculated wave field intensity on each atomic layer from the top layer $\left(\mathrm{L}_{1}\right)$ to the fourth layer $\left(\mathrm{L}_{4}\right)$ while changing the glancing angle from $0.5^{\circ}$ to $6.5^{\circ}$. The intensity generally weakens with increasing depth toward $\mathrm{L}_{4}$; however, intensity anomalies on atomic layers $\mathrm{L}_{1}-\mathrm{L}_{4}$ occur similarly under all Bragg conditions. An intensity peak is observed in the low-angle side, and an intensity minimum is observed in the high-angle side of each Bragg angle. The former corresponds to the wave-field 


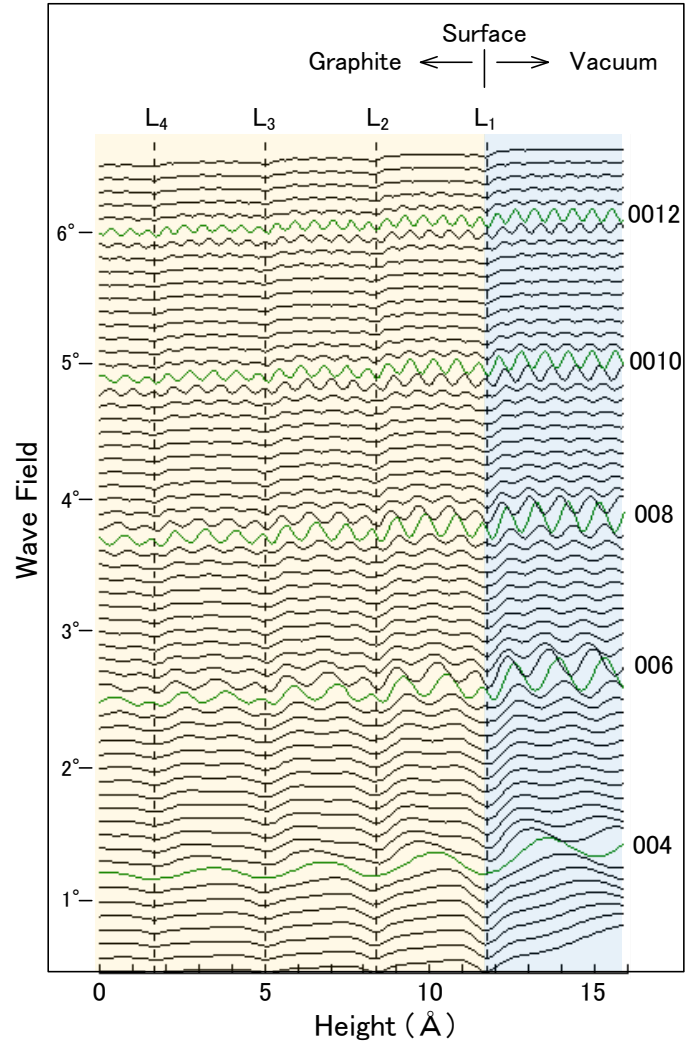

FIG. 5. Calculated wave fields in the region from vacuum to the fourth layer $\mathrm{L}_{4}$ of graphite while changing the glancing angle from $0.5^{\circ}$ to $6.5^{\circ}$. The broken lines indicate the depth positions from the first layer $\mathrm{L}_{1}$ (surface) to the fourth layer $\mathrm{L}_{4}$. Bragg conditions are indicated by the reflection indices on the right side.

intensity maxima riding on the atomic planes, whereas the latter corresponds to those on the interatomic planes. These anomalous behaviors drastically change depending on the surface structure, and the details will be reported elsewhere.

Figures 7(a) and 7(b) show the calculated and experimental BRAES profiles of C-KLL, respectively. Fig-

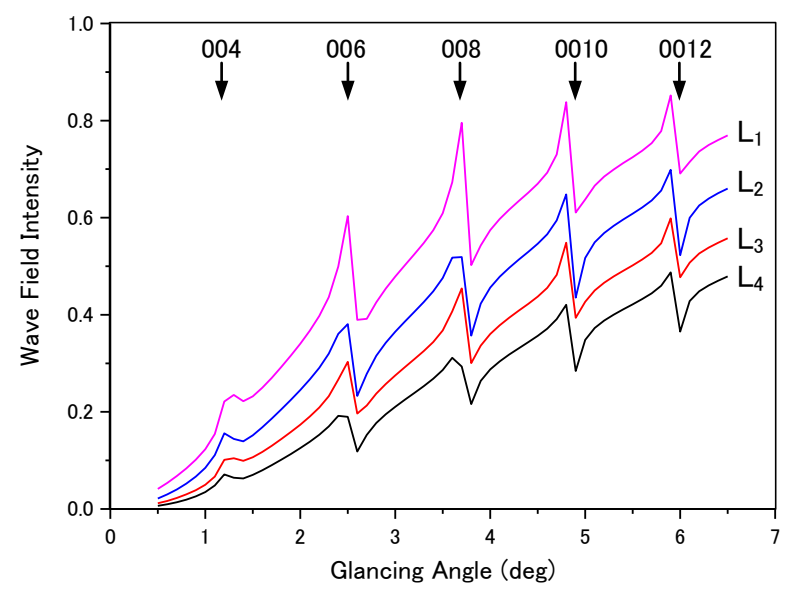

FIG. 6. Wave field intensities on each atomic layer from the first layer $\mathrm{L}_{1}$ to the fourth layer $\mathrm{L}_{4}$ as a function of the glancing angle.

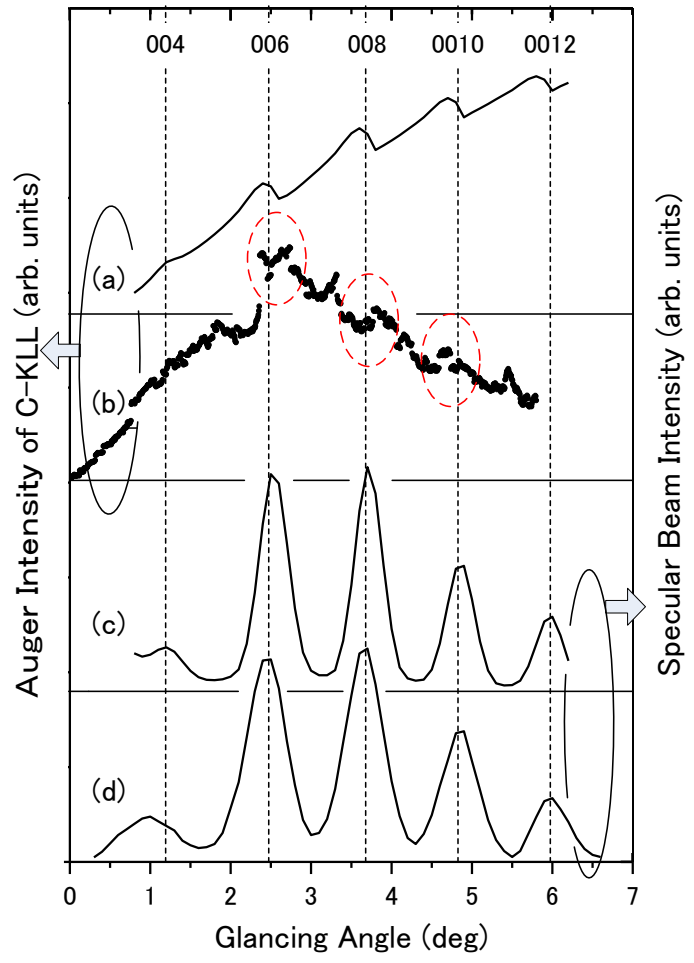

FIG. 7. (a) and (b) are the calculated and experimental BRAES profiles, respectively. (c) and (d) are the calculated and experimental rocking curves, respectively.

ures $7(\mathrm{c})$ and $7(\mathrm{~d})$ show the calculated and experimental rocking curves of the specularly reflected beam, respectively. The results of Figs. 7(a) and 7(c) are calculated based on the results of Fig. 6 and Fig. 4(c), respectively, by considering the divergent effect, which makes the BRAES and the rocking curve mild. It is assumed that the Auger excitation probability at an atomic layer is proportional to the wave field intensity at the layer. The calculated BRAES of Fig. 7(a) is the sum of Auger intensities emitted from the top four layers $\left(\mathrm{L}_{1}-\mathrm{L}_{4}\right)$, by considering the exponential decay due to the escape depth of about $5 \AA$ for C-KLL Auger electron with a kinetic energy of about $270 \mathrm{eV}$ [17].

The experimental BRAES profile is sensitive to the cleaved surface conditions, including bending and surface steps. This high surface sensitivity comes from the short escape depth of Auger electrons. Although the signal-tonoise ratio of the experimental BRAES profile of Fig. 7(b) is not good, the intensity anomalies indicated by dotted ellipses at the Bragg conditions 0006,008 , and 0010 almost agree with the calculated profile. There may be some ambiguities of the top-layer height around many step edges due to cleavage treatment because of the weak van der Waals interaction. This is considered to prevent the good agreement between the experimental and the calculated anomalies in BRAES profiles. For Bragg conditions such as 006,008 , and 0010 , the periods between the intensity maximum and minimum of wave-field are very short such as $0.6 \AA, 0.4 \AA$, and $0.3 \AA$, respectively. Then, the shape of the anomalous peak on the BREAS profile is sensitive to change of the top-layer height. Ambiguity of the top-layer height is considered to modulate the shape of the anomalous peak. 
In spite of the monotonous increase of the calculated BRAES background, the experimental profile has a broad peak around $3^{\circ}$, and it gradually decreases with the glancing angle, which may be mainly caused due to the slight deviation of the incident beam axis from the center of the sample surface. Since CMA for an Auger detector focuses on the center of the sample surface, slight deviation of the incident beam from that point will influence the BRAES profile more sensitively than the rocking curve. For the rocking curves, experimental and calculated peaks agree well with not only the angle but also the relative intensity. Only a small difference is seen at angles lower than $1^{\circ}$. This is considered to be mainly due to surface steps introduced by cleavage.

\section{CONCLUSION}

We observed the mosaic-like RHEED pattern on the HOPG (Panasonic Graphite Crystal) surface. For this polycrystalline material, the rocking curve analysis showed that the one-beam dynamical approach is effec- tive for the analysis of the interlayer distance of HOPG. The calculated result based on the ideal surface structure without relaxation can accurately reproduce the obtained experimental result. The BRAES profile was very sensitive to the surface conditions, such as a stepped surface after cleaving treatment, due to the very shallow escape depth of the Auger electrons. Auger intensity anomalies were detected under the Bragg conditions, which are related to the behavior of the wave field on the atomic layers. The wave field was sensitive to the surface structure, especially for higher-order Bragg conditions. The results of this behavior will be reported elsewhere. Auger excitation by wave field can be used to perform surface sensitive structural analysis.

\section{ACKNOWLEDGMENTS}

This study is supported by JSPS KAKENHI Grant Number 16K06816 and the Cooperative Research Program of Network Joint Research Center for Materials and Devices.
[1] Y. Horio and A. Ichimiya, Physica B 117-118, 792 (1983).

[2] A. Ichimiya and Y. Takeuchi, Surf. Sci. 128, 343 (1983).

[3] Y. Horio and A. Ichimiya, Surf. Sci. 164, 589 (1985).

[4] Y. Horio, Jpn. J. Appl. Phys. 37, L164 (1998).

[5] Y. Horio and D. Sakai, Jpn. J. Appl. Phys. 48, 066501 (2009).

[6] Y. Horio, Y. Takakuwa, and S. Ogawa, Surf. Interface Anal. 46, 1165 (2014).

[7] H. Marten and G. Meyer-Ehmsen, Acta Crystallogr. A 44, 853 (1988).

[8] T. Kawamura and P. A. Maksym, Surf. Sci. 601, 822 (2007).

[9] Y. Fukaya, S. Entani, S. Sakai, I. Mochizuki, K. Wada, T. Hyodo, and S. Shamoto, Carbon 103, 1 (2016).

[10] Y. Fukaya, I. Mochizuki, M. Maekawa, K. Wada, T. Hyodo, I. Matsuda, and A. Kawasuso, Phys. Rev. B 88,
205413 (2013)

[11] Y. Fukaya, I. Matsuda, B. Feng, I. Mochizuki, T. Hyodo, and S. Shamoto, 2D Mater. 3, 035019 (2016).

[12] S. Miyake, K. Hayakawa, and R. Miida, Acta Crystallogr. A 24, 182 (1968).

[13] T. Yamanaka, T. Hanada, and S. Ino, Phys. Rev. Lett. 75, 669 (1995).

[14] Y. Horio, Y. Takakuwa, S. Ogawa, and K. Abe, e-J. Surf. Sci. Nanotech. 14, 92 (2016).

[15] A. Ichimiya, Jpn. J. Appl. Phys. 22, 176 (1983).

[16] P. A. Doyle and P. S. Turner, Acta Crystallogr. A 24, 390 (1968).

[17] D. Briggs and M. P. Seah, Practical Surface Analysis by Auger and X-ray Photoelectron Spectroscopy, 2nd ed. (John Wiley, Chichester, 1990). 